\title{
FOURIER INTEGRAL OPERATORS AND NONLINEAR WAVE EQUATIONS
}

\author{
CHRISTOPHER D. SOGGE \\ Department of Mathematics, Johns Hopkins University \\ Baltimore, Maryland 21218, U.S.A. \\ E-mail:sogge@jhu.edu
}

1. Introduction. The purpose of this paper is to indicate how one can use Fourier integral operators and techniques from harmonic analysis to prove a sharp global existence theorem for nonlinear wave equations with power-type nonlinearities. Specifically, if $f, g \in$ $C_{0}^{\infty}\left(\mathbb{R}^{n}\right), n \geq 2$ are fixed, then one always has global existence for equations of the form

$$
\left\{\begin{array}{l}
\square u= \pm|u|^{p}, \quad(t, x) \in \mathbb{R}_{+} \times \mathbb{R}^{n}=\mathbb{R}_{+}^{1+n} \\
u(0, x)=\varepsilon f(x), \quad \partial_{t} u(0, x)=\varepsilon g(x),
\end{array}\right.
$$

provided that $\varepsilon>0$ is small and $p>1$ satisfies

$$
(n-1) p^{2}-(n+1) p-2>0 .
$$

Here $\square=\partial_{t}^{2}-\Delta_{x}$ denotes the D'Alembertian. Also, one can replace $\pm|u|^{p}$ by $F_{p}(u)$ if $F_{p}^{(j)}(u)=O\left(|u|^{p-j}\right), j=0,1$. Results for superconformal nonlinearities were already known (see, e.g. [11]), so in what follows we shall also always assume that $p$ is smaller than the conformal power, that is,

$$
p<(n+3) /(n-1) .
$$

This existence theorem is due to V. Georgiev, H. Lindblad and the author [3]. Many earlier partial results have been known for some time. The first, and most important, goes back to John [9] who solved the problem when $n=3$. He showed that, as in (1.2), global solutions always exist for small $\varepsilon$ if $p>1+\sqrt{2}$, while, conversely, if $p<1+\sqrt{2}$ there can be blowup even for small $\varepsilon>0$. Based on this, and his related work [23] Strauss conjectured in [24] that the aforementioned $n$-dimensional version should hold. This was quickly verified by Glassey [5] when $n=2$. In the other direction Sideris [17] showed that even for small $\varepsilon$ solutions blowup if $p$ does not satisfy (1.2). More recently, Zhou

1991 Mathematics Subject Classification: Primary 35; Secondary 42.

The author was supported in part by the NSF.

The paper is in final form and no version of it will be published elsewhere. 
[27] showed that Strauss' conjecture holds when $n=4$, and Lindblad and the author [12] showed that it holds when $n \leq 8$, and also for any $n$ under the assumption of spherical symmetry. The latter result was also obtained independently for odd $n$ by Kubo [10].

The proof of the aforementioned existence results hinges on a certain weighted version of an inequality of Strichartz [25] for the inhomogeneous wave equation. Before stating the new results, let us explain what happens in the unweighted case.

If we consider the inhomogeneous wave equation

$$
\left\{\begin{array}{l}
\square w=F, \quad(t, x) \in \mathbb{R}_{+}^{1+n} \\
0=w(0, x)=\partial_{t} w(0, x),
\end{array}\right.
$$

and if $n \geq 2$, then the main estimate from [25] is that

$$
\|w\|_{L^{q}\left(\mathbb{R}_{+}^{1+n}\right)} \leq C_{n}\|F\|_{L^{q /(q-1)}\left(\mathbb{R}_{+}^{1+n}\right)}, \quad q=2(n+1) /(n-1) .
$$

Notice that the inequality involves conjugate exponents and that it is scale invariant since

$$
(n+1)(1 / q-(q-1) / q)=2, \quad q=2(n+1) /(n-1) .
$$

Inequality (1.5) is a hyperbolic version of the classical inequality of Hardy, Littlewood and Sobolev for the Laplacian

$$
\|\phi\|_{L^{q}\left(\mathbb{R}^{d}\right)} \leq C_{p, q, d}\|\Delta \phi\|_{L^{q}\left(\mathbb{R}^{d}\right)}, \phi \in \mathcal{S}\left(\mathbb{R}^{d}\right), 1<p<q<\infty, d(1 / p-1 / q)=2 .
$$

Inequality (1.6) extends to some exponents off of the line of duality; however, Harmse [6] showed that if $d=n+1$ and if we replace $\Delta$ by $\square$, then the inequality holds if and only if $\min (1 / p-1 / 2,1 / 2-1 / q) \in(1 / 2 n, 1 /(n+1)]$. This restriction in part makes problems like (1.1) difficult to analyze.

It turns out that much more favorable weighted inequalities hold for the Laplacian as opposed to the D'Alembertian, even if one considers dual exponents. Indeed, results of Jerison and Kenig [8] and Müller [14] yield

$$
\begin{aligned}
& \left\||x|^{\tau-2+2 d(1 / 2-1 / q)} \phi\right\|_{L^{q}\left(\mathbb{R}^{d}\right)} \leq C_{n, \delta}\left\||x|^{\tau} \Delta \phi\right\|_{L^{q /(q-1)}\left(\mathbb{R}^{d}\right)}, \\
& \quad \text { if } \phi \in C_{0}^{\infty}\left(\mathbb{R}^{d} \backslash 0\right), \operatorname{dist}(\tau, \mathbb{Z}+(d-2) / 2) \geq \delta>0, \text { and } 2 \leq q \leq 2 d /(d-2) .
\end{aligned}
$$

Like the preceding ones, this inequality is scale invariant.

The inequality that is used to establish the existence results for (1.1) involves what is, in some sense, an optimal extension of [8] to the hyperbolic setting. Here the weights should evidently involve the Lorentzian distance from the origin as opposed to the one given by the Euclidean metric. Since the former is well defined inside the light cone $\Gamma_{+}=\left\{(t, x) \in \mathbb{R}_{+}^{1+n}: t^{2}-|x|^{2}>0\right\}$ it is natural to consider solutions of (1.4) which are supported in this set. Also, since generic solutions of (1.4) only decay like $O\left(t^{-(n-1) / 2}\right)$ as $t \rightarrow+\infty$, even if $F \in C_{0}^{\infty}\left(\Gamma_{+}\right)$, one could not hope for the variant of (1.7) for the D'Alembertian to hold for arbitrarily large $\tau$.

Keeping these things in mind, let us state the main inequality from [3]:

THEOREM 1.1. Let $n \geq 2$ and assume that $F \in \mathcal{S}$ satisfies

$$
F(t, x)=0 \quad \text { if }|x|>t-1 .
$$


Then if $w$ is the solution of (1.4), and if $\varepsilon>0$ there is a constant $C=C(\varepsilon, n, q)$ so that

$$
\begin{array}{r}
\left\|\left(t^{2}-|x|^{2}\right)^{-1 / 2+n(1 / 2-1 / q)-\varepsilon} w\right\|_{L^{q}\left(\mathbb{R}_{+}^{1+n}\right)} \leq C\left\|\left(t^{2}-|x|^{2}\right)^{1 / q+\varepsilon} F\right\|_{L^{q /(q-1)}\left(\mathbb{R}_{+}^{1+n}\right)}, \\
\quad \text { if } 2 \leq q \leq 2(n+1) /(n-1) .
\end{array}
$$

With $d=n+1$ being the space-time dimension (1.9) corresponds, up to an endpoint, to (1.7) with $\tau=1 / q$. We also remark that (1.9) is essentially scale invariant. The dilation invariant version would involve $\varepsilon=0$, in which case we could also weaken the support assumptions to $F(t, x)=0$ if $|x| \geq t$. Unfortunately, this version can never hold, even if we assume that $F$ is supported in a fixed compact set since the norms in the left would, in general, be infinite since we could choose $F$ so that $w$ is bounded below by $t^{-(n-1) / 2}$ for large $t$ in, say, $\{(t, x): 1 \leq t \leq 2\}$. This explains one of the differences between (1.9) and its elliptic counterpart (1.7) that we mentioned earlier. We also remark that when $q=2(n+1) /(n-1)$, (1.9) should be thought of as a weighted version of (1.5). By interpolating with the latter, one sees that the inequality extends to one involving weights with a larger range of powers; however, the ones in (1.9) are the most difficult to prove and are what are needed to prove optimal existence results for (1.1).

To see this, let us set $u_{-1} \equiv 0$ and then define $u_{m}, m=0,1,2, \ldots$ recursively by requiring that $\square u_{m}=F_{p}\left(u_{m-1}\right), u_{m}(0, x)=\varepsilon f(x), \partial_{t} u_{m}(0, x)=\varepsilon g(x)$, where $f$ and $g$ are as in (1.1). We clearly may assume without loss of generality that they are supported in the unit ball. Notice that we can split $u_{m}=u_{0}+w_{m}$, where $\square u_{0}=0$ with data $(f, g)$ and $\square w_{m}=F_{p}\left(u_{m-1}\right)$ with zero data at $t=0$. To proceed, note that if $p$ satisfies our assumptions (1.1), (1.3) then we can always chose $\gamma$ satisfying

$$
1 / p(p+1)<\gamma<-1 / 2+n(1 / 2-1 /(p+1)) .
$$

If we shift the time coordinate and if $w$ and $F$ are as in (1.4), then (1.9) clearly yields

$$
\begin{array}{r}
\left\|\left(1+\left.\left|t^{2}-\right| x\right|^{2} \mid\right)^{\gamma} w\right\|_{L^{p+1}\left(\mathbb{R}_{+}^{1+n}\right)} \leq C_{\gamma, p}\left\|\left(1+\left.\left|t^{2}-\right| x\right|^{2} \mid\right)^{\gamma / p} F\right\|_{L^{(p+1) / p}\left(\mathbb{R}_{+}^{1+n}\right)}, \\
\text { if } F(t, x)=0,|x| \geq t+1 .
\end{array}
$$

If we replace $w$ by $u_{0}$ in the left, then the resulting quantity is $O(\varepsilon)$, in view of our earlier discussion, since $u_{0}=O\left([(1+|t-r|) t]^{-(n-1) / 2}\right)$. Furthermore, if we apply the preceding inequality to $w=w_{m}$ we conclude that

$$
A_{m} \leq C \varepsilon+C A_{m-1}^{p}, \quad m \geq 1,
$$

if $A_{m}=\left\|\left(1+\left.\left|t^{2}-\right| x\right|^{2} \mid\right)^{\gamma} u_{m}\right\|_{L^{p+1}\left(\mathbb{R}_{+}^{1+n}\right)}$. Since $A_{0}=O(\varepsilon)$, this implies that $A_{m} \leq C_{0} \varepsilon$ for some uniform constant if $\varepsilon>0$ in (1.1) is small enough. Similar arguments then give that $2 B_{m} \leq B_{m-1}$ if $B_{m}=\left\|\left(1+\left.\left|t^{2}-\right| x\right|^{2} \mid\right)^{\gamma}\left(u_{m}-u_{m-1}\right)\right\|_{L^{p+1}\left(\mathbb{R}_{+}^{1+n}\right)}$ and $\varepsilon$ is small. Using these two bounds it is not hard to see that $u_{m}$ must converge to a (weak) solution of (1.1) verifying $\left(1+\left.\left|t^{2}-\right| x\right|^{2} \mid\right)^{\gamma} u \in L^{p+1}\left(\mathbb{R}_{+}^{1+n}\right)$. These arguments also show that this solution is unique.

Let us conclude this section by sketching the proof of the original, unweighted estimate of Strichartz (1.5) since this will provide a model for (1.9). For this, let $\chi_{+}^{z}(t)$ denote the analytic family of distributions $t_{+}^{z} / \Gamma(1+z)$, where if $\operatorname{Re} z>-1$, the distribution equals 
$t^{z} / \Gamma(1+z)$ for $t>0$ and 0 otherwise. We then set

$$
w_{z}(t, x)=(2 \pi)^{-n} e^{z^{2}} \iint e^{i x \cdot \xi} \sin ((t-s)|\xi|) \chi_{+}^{z}(t-s) \hat{F}(s, \xi)|\xi|^{-1-z} d \xi d s,
$$

where $\hat{F}$ denotes the spatial Fourier transform of $F$. Since $w_{0}=w$, if we apply Stein's analytic interpolation theorem [22], we conclude that it suffices to show that if $F$ is supported in $\mathbb{R}_{+}^{1+n}$ then

$$
\begin{aligned}
\left\|w_{z}\right\|_{L^{2}\left(\mathbb{R}_{+}^{1+n}\right)} \leq C\|F\|_{L^{2}\left(\mathbb{R}_{+}^{1+n}\right)}, & \operatorname{Re} z=-1, \\
\left\|w_{z}\right\|_{L^{\infty}\left(\mathbb{R}_{+}^{1+n}\right)} \leq C\|F\|_{L^{1}\left(\mathbb{R}_{+}^{1+n}\right)}, & \operatorname{Re} z=(n-1) / 2 .
\end{aligned}
$$

The first estimate follows from an application of Plancherel's theorem and Euler's formula since

$$
e^{z^{2}} \int_{-\infty}^{\infty} e^{i t \tau} \chi_{+}^{z}(t) d t=O(1), \operatorname{Re} z=-1
$$

The $L^{\infty}$ estimate is also elementary. It just follows from the fact that

$$
e^{z^{2}}|t|^{(n-1) / 2} \int_{\mathbb{R}^{n}} e^{i x \cdot \xi+i t|\xi|}|\xi|^{-1-z} d \xi=O(1), \operatorname{Re} z=(n-1) / 2 .
$$

2. $L^{2}$ estimates for nondegenerate Fourier integral operators: A review. The proof of (1.9) splits into two parts. The first relies on standard $L^{2}$ estimates for Fourier integral operators and a simple argument exploiting the Lorentz invariance of the weights. The other step in the proof requires estimates for degenerate Fourier integral operators which are similar to those arising in the Goursat problem. We shall postpone the statement of these until they are needed, but, fortunately, their proof just involves adapting the proof of the bounds for nondegenerate operators in a straightforward way.

In this section let us review the classical $L^{2}$ estimates which we shall use in the easier half of the proof. We shall consider zero-order local Fourier integral operators of the form

$$
(W f)(x)=\iint e^{i x \cdot \xi+i \varphi(y, \xi)} a(y, \xi) f(y) d \xi d y .
$$

Here $\varphi$ is assumed to be real, homogeneous of degree one in $\xi$ and in $C^{\infty}\left(\mathbb{R}^{n} \times \mathbb{R}^{n} \backslash 0\right)$. We assume that the symbol satisfies

$$
a(y, \xi)=0, \quad(y, \xi) \notin K \times \Gamma
$$

where $K \subset \mathbb{R}^{n}$ is a fixed compact set and $\Gamma$ is a fixed conic subset of $\mathbb{R}^{n} \backslash 0$. We also assume that the symbol is zero-order, meaning here that

$$
\left|(\partial / \partial y)^{\alpha} a(y, \xi)\right| \leq C_{\alpha}
$$

for all multi-indices $\alpha$.

The estimate we require then is the following

Proposition 2.1. If a satisfies (2.1) and (2.2) and if

$$
\operatorname{det}\left(\partial^{2} \varphi / \partial y_{j} \partial \xi_{k}\right) \neq 0, \quad(y, \xi) \in K \times \Gamma,
$$


then

$$
\|W f\|_{L^{2}\left(\mathbb{R}^{n}\right)} \leq C\|f\|_{L^{2}\left(\mathbb{R}^{n}\right)},
$$

where, if $\varphi$ is fixed, the constant depends only on finitely many of the bounds in (2.2).

This estimate, in different forms, goes back to Hörmander [7] and Eskin [2]. As was shown in [7], it implies the seemingly more general fact that zero-order Fourier integral operators which are locally a canonical graph are (locally) bounded on $L^{2}$.

Pr o of. The first thing to notice is that, by Plancherel's theorem,

$$
\|W f\|_{L^{2}}^{2}=(2 \pi)^{-n} \int_{\mathbb{R}^{n}}|(\tilde{W} f)(\xi)|^{2} d \xi,
$$

where

$$
\tilde{W} f(\xi)=\int e^{i \varphi(y, \xi)} a(y, \xi) f(y) d y .
$$

Therefore, by duality, (2.4) is equivalent to the estimate

$$
\left\|\tilde{W}^{*} g\right\|_{L^{2}(d y)} \leq C\|g\|_{L^{2}},
$$

where $\tilde{W}^{*}$ denotes the adjoint operator.

Next, notice that by the mean value theorem and homogeneity

$$
\nabla_{y}(\varphi(y, \xi)-\varphi(y, \eta))=\left(\frac{\partial^{2} \varphi(y, \xi)}{\partial y \partial \xi}\right)(\xi-\eta)+r(y, \eta, \xi)
$$

where, by compactness,

$$
|r(y, \xi, \eta)| \leq C|\xi /| \xi|-\eta /| \eta|| \cdot|\xi-\eta|,
$$

uniformly on the support of $a$. If we break up the operator into a finite number of pieces, then we conclude from (2.3) that we may assume that

$$
\left|\nabla_{y}(\varphi(y, \xi)-\varphi(y, \eta))\right| \geq\left|\left(\frac{\partial^{2} \varphi(y, \xi)}{\partial y \partial \xi}\right)(\xi-\eta)\right| / 2
$$

when $(y, \xi),(y, \eta) \in K \times \Gamma$. If we make another decomposition, we find that we see that there is no loss of generality in strengthening this assumption to

$$
\left|\left(\partial / \partial y_{1}\right)(\varphi(y, \xi)-\varphi(y, \eta))\right| \geq c|\xi-\eta|,
$$

on the support of $a$ for some uniform $c>0$.

To use this, we note that

$$
\left\|\tilde{W}^{*} g\right\|_{L^{2}}^{2}=\iint K(\xi, \eta) g(\xi) \overline{g(\eta)} d \xi d \eta
$$

where

$$
K(\xi, \eta)=\int e^{i(\varphi(y, \xi)-\varphi(y, \eta))} a(y, \xi) \overline{a(y, \eta)} d y
$$

Clearly, by (2.1),

$$
K=O(1)
$$

Also, since

$$
L(y, D) e^{i(\varphi(y, \xi)-\varphi(y, \eta))}=e^{i(\varphi(y, \xi)-\varphi(y, \eta))}
$$


where $L=\left(i \partial / \partial y_{1}(\varphi(y, \xi)-\varphi(y, \eta))\right)^{-1} \partial / \partial y_{1}$, if we integrate by parts we obtain

$$
K(\xi, \eta)=\int e^{i(\varphi(y, \xi)-\varphi(y, \eta))}\left(L^{*}\right)^{N} a(y, \xi) \overline{a(y, \eta)} d y,
$$

for a given $N=1,2,3, \ldots$. Since (2.1) and (2.6) imply that for $|\xi-\eta| \geq 1$

$$
\left(L^{*}\right)^{N} a(y, \xi) \overline{a(y, \eta)}=O\left(|\xi-\eta|^{-N}\right),
$$

we conclude that $K=O\left(|\xi-\eta|^{-N}\right)$ for any $N$ if $|\xi-\eta| \geq 1$. If we combine this with our earlier bound, we conclude that

$$
K(\xi, \eta)=O\left((1+|\xi-\eta|)^{-N}\right), \quad N=1,2,3, \ldots
$$

If we take $N=n+1$, then an application of Young's inequality along with (2.7) yields (2.5) which finishes the proof.

3. Lorentz invariance and a variation on Strichartz's estimate. Let us start out by seeing that, using Proposition 2.1, we can easily adapt the proof of the unweighted Strichartz estimates to obtain the special case of (1.9) where the norms are only taken over the "middle" of the light cone. To be more specific, if

$$
\Gamma_{\text {mid }}=\left\{(t, x) \in \mathbb{R}_{+}^{1+n}:|x|<t / 2\right\},
$$

then we have the following

Proposition 3.1. Assume that $F=0$ if $t^{2}-|x|^{2} \leq 1$ and that $w$ is the solution of the inhomogeneous wave equation (1.4) with zero data. Then if $T \geq 2$,

$$
\begin{aligned}
& \left\|\left(t^{2}-|x|^{2}\right)^{-1 / 2+n(1 / 2-1 / q)} w\right\|_{L^{q}\left(\left\{(t, x) \in \Gamma_{\text {mid }}: T / 2 \leq t \leq T\right\}\right)} \\
& \quad \leq C(\log T)^{1 / q}\left\|\left(t^{2}-|x|^{2}\right)^{1 / q} F\right\|_{L^{q /(q-1)}}, \quad 2 \leq q \leq 2(n+1) /(n-1) .
\end{aligned}
$$

Notice that, except for the restriction $(t, x) \in \Gamma_{\text {mid }}$, the bounds here are stronger than those in (1.5). Also, by Stein's interpolation theorem, to prove (3.1), it suffices to prove the inequality for the endpoints where $q=2$ or $q=2(n+1) /(n-1)$.

The first step in proving the inequality for $q=2(n+1) /(n-1)$ is to notice that the unweighted version, (1.5), yields (3.1) if we assume that $F(t, x)=0$ when $t-|x| \leq T / 10$, say. Under this assumption the $L^{2}$ estimate is also trivial since

$$
\|w\|_{L^{2}(\{(t, x): T / 2 \leq t \leq T\})} \leq C T^{2}\|F\|_{L^{2}\left(\mathbb{R}_{+}^{1+n}\right)} .
$$

Hence, in proving (3.1), we can also assume that $t-|x| \leq T / 10$ in the support of $F$.

For the next easy reduction, we need to recall that $w=E_{+} * F$, where

$$
E_{+}=\pi^{(1-n) / 2} / 2 \cdot \chi_{+}^{-(n-1) / 2}\left(t^{2}-|x|^{2}\right) .
$$

Since $E_{+}(t-s, x-y)=O\left(T^{-(n-1)}\right)$ if $T / 2 \leq t \leq T,|x| \leq t / 2$, and $|y| \leq T / 10,(3.1)$ trivially holds if $|y| \leq T / 10$ in the support of $F$. If we combine this with the preceding step we conclude that, to prove (3.1), we may assume that

$$
F(s, y)=0 \text { if }|y| \leq T / 10 \text { or } s-|y| \notin[1 / 2 T, T / 10] .
$$

The lower bound on $s-|y|$ comes from our assumption that $F=0$ when $s^{2}-|y|^{2}<1$. 
To proceed, we recall that

$$
(2 \pi)^{n} w(t, x)=\frac{1}{2 i} \sum_{ \pm} \int_{0}^{t} \iint e^{i(x-y) \cdot \xi \pm i(t-s)|\xi|} F(s, y) \frac{d \xi}{|\xi|} d y d s .
$$

Note that the absolute value of the $\xi$-gradient of the phase function satisfies

$$
\begin{aligned}
& |x-y \pm(t-s) \xi /| \xi|| \geq c T, \text { if }|\xi /| \xi|\mp y /| y|| \leq c, \\
& \qquad \text { if }(t, x) \in \Gamma_{\text {mid }}, T / 2 \leq t \leq T,(s, y) \in \operatorname{supp} F,
\end{aligned}
$$

for some uniform constant $c>0$. Consequently, we can integrate by parts to see that we can write

$$
w(t, x)=\sum_{ \pm} \iiint_{|\xi| \geq 1 / T} e^{i(x-y) \cdot \xi \pm i(t-s)|\xi|} a_{ \pm}(y, \xi) F(s, y) \frac{d \xi}{|\xi|} d y d s+\left(R_{ \pm} F\right)(t, x),
$$

where

$$
a_{ \pm}(y, \xi)=0 \text { if }|y /| y|\mp \xi /| \xi|| \leq c / 2 \text {, and } \partial_{y}^{\alpha} a_{ \pm}=O\left(T^{-|\alpha|}\right) \forall \alpha,
$$

and where $R_{ \pm}$is an integral operator with a kernel which is $O\left(T^{-(n-1)}\right)$. Consequently, if we replace $w$ by $R_{ \pm} F$, the variant of (3.1) holds under our assumption (3.2), and therefore it suffices to show that for $q=2$ or $q=2(n+1) /(n-1)$

$$
\begin{aligned}
T^{-1+2 n(1 / 2-1 / q)}\left\|W_{ \pm} F\right\|_{L^{q}\left(\left\{(t, x) \in \mathbb{R}_{+}^{1+n}: T / 2 \leq t \leq T\right\}\right)} & \\
& \leq C(\log T)^{1 / q}\left\|\left(t^{2}-|x|^{2}\right)^{1 / q} F\right\|_{L^{q /(q-1)}},
\end{aligned}
$$

assuming that $F$ is as in (3.2), with

$$
\left(W_{ \pm} F\right)(t, x)=\iiint_{|\xi| \geq 1 / T} e^{i(x-y) \cdot \xi \pm i(t-s)|\xi|} a_{ \pm}(y, \xi) F(s, y) \frac{d \xi}{|\xi|} d y d s .
$$

To prove this inequality, we first notice that if we write $(s, y)=(\tau+|y|, y)$ and apply Hölder's inequality we obtain

$$
\times\left(\int_{1 / 2 T}^{T / 10}\left|\iint_{|\xi| \geq 1 / T} e^{i(x-y) \cdot \xi \pm i(t-\tau-|y|)|\xi|} a_{ \pm}(y, \xi) \tau^{1 / q} F(\tau+|y|, y) \frac{d \xi}{|\xi|} d y\right|^{q /(q-1)} d \tau\right)^{(q-1) / q}
$$

Using Plancherel's theorem, we conclude that

$$
\begin{aligned}
& \int\left|W_{ \pm} F(t, x)\right|^{2} d x \\
& \quad \leq C \log T \int\left(\int\left|\iint_{|\xi| \geq 1 / T} e^{i(x-y) \cdot \xi \pm i|y| \cdot|\xi|} a_{ \pm}(y, \xi) \tau^{1 / 2} F(\tau+|y|, y) \frac{d \xi}{|\xi|} d y\right|^{2} d x\right) d \tau
\end{aligned}
$$

Let

$$
\varphi_{ \pm}(y, \xi)=|y| \cdot|\xi| \pm y \cdot \xi
$$

be the phase function in the preceding Fourier integral. Since

$$
\operatorname{det}\left(\partial^{2} \varphi_{ \pm} / \partial y_{j} \partial \xi_{k}\right)= \pm y /|y| \cdot \xi /|\xi|-1 \neq 0 \text { on } \operatorname{supp} a_{ \pm},
$$


we can apply Proposition 2.1 and a change of scale argument to conclude that

$$
\begin{aligned}
\int \mid \iint_{|\xi| \geq 1 / T} e^{i(x-y) \cdot \xi \pm i|y| \cdot|\xi|} a_{ \pm}(y, \xi) \tau^{1 / 2} F(\tau+|y|, y) & \left.\frac{d \xi}{|\xi|} d y\right|^{2} d x \\
& \leq C T^{2} \int\left|\tau^{1 / 2} F(\tau+|y|, y)\right|^{2} d y .
\end{aligned}
$$

Combining this with the preceding inequality yields (3.4) when $q=2$.

To prove (3.4) for the other endpoint, $q=2(n+1) /(n-1)$, we need to also use the interpolation argument that was used to establish the unweighted Strichartz inequality (1.5). Specifically, if we let

$$
\begin{aligned}
& \left(W_{ \pm}^{z} F\right)(t, x) \\
& \quad=e^{z^{2}} \iiint_{|\xi| \geq 1 / T} e^{i(x-y) \cdot \xi \pm i(t-s) \cdot|\xi|} \chi_{+}^{z}(t-s)|\xi|^{-1-z} a_{ \pm}(y, \xi) F(s, y) d \xi d y d s
\end{aligned}
$$

then it suffices to show that

$$
\begin{aligned}
\left\|W_{ \pm}^{z} F\right\|_{L^{\infty}\left(\left\{(t, x) \in \mathbb{R}_{+}^{1+n}: T / 2 \leq t \leq T,|x| \leq t / 2\right\}\right)} & \leq C\|F\|_{L^{1}}, \operatorname{Re} z=(n-1) / 2, \\
T\left\|W_{ \pm}^{z} F\right\|_{L^{2}\left(\left\{(t, x) \in \mathbb{R}_{+}^{1+n}: T / 2 \leq t \leq T\right\}\right)} & \leq C(\log T)^{1 / 2}\left\|\left(t^{2}-|x|^{2}\right)^{1 / 2} F\right\|_{L^{2}}, \operatorname{Re} z=-1,
\end{aligned}
$$

assuming in both cases that $F$ satisfies (3.2) and that $T$ is large.

Note that $t-s \approx T$ in the support of the integrand in (3.7) if $|x| \leq t / 2$. Because of this the $L^{\infty}$ estimate follows immediately from (1.12). Since in (3.7) $\chi_{+}^{z}(t-s)|\xi|^{-1-z}=$ $O(1 / T), \operatorname{Re} z=-1$, one obtains the $L^{2}$ estimate by repeating the arguments which gave (3.4) for $q=2$.

Let us now see how Proposition 3.1 yields the following special case of our main estimate (1.9).

Proposition 3.2. Fix $T \geq 2$ and assume that $F(t, x)$ vanishes if $|x|>t-1$ or if $t \leq T / 10$. Then, if $w$ is the solution of the inhomogeneous wave equation (1.4), there is a constant $C$, depending only on the dimension $n \geq 2$, so that

$$
\begin{aligned}
& \left\|\left(t^{2}-|x|^{2}\right)^{-1 / 2+n(1 / 2-1 / q)} w\right\|_{L^{q}\left(\left\{(t, x) \in \mathbb{R}_{+}^{1+n}: T / 2 \leq t \leq T\right\}\right)} \\
& \quad \leq C(\log T)^{2 / q}\left\|\left(t^{2}-|x|^{2}\right)^{1 / q} F\right\|_{L^{q /(q-1)}}, \quad 2 \leq q \leq 2(n+1) /(n-1) .
\end{aligned}
$$

We first note that to prove (3.8) we may assume that $T$ is large since the estimate for small fixed $T$ is a simple consequence of (1.5) and the support assumptions on $F$. The next thing to notice is that (3.8) follows from Proposition 3.1 and the dyadic estimates

$$
\begin{aligned}
& \left\|\left(t^{2}-|x|^{2}\right)^{-1 / 2+n(1 / 2-1 / q)} w\right\|_{L^{q}\left(\left\{(t, x): T / 2 \leq t \leq T, 2^{-k-1} T \leq t-|x| \leq 2^{-k} T\right\}\right)} \\
\leq & C(\log T)^{1 / q}\left\|\left(t^{2}-|x|^{2}\right)^{1 / q} F\right\|_{L^{q /(q-1)}}, 2 \leq q \leq 2(n+1) /(n-1), 0 \leq k \leq \log _{2} T .
\end{aligned}
$$

Uniform bounds of this type yield (3.8) if one raises both sides to the $q$-th power and sums over $k$.

The key observation behind the proof of (3.9) is the following simple geometric fact (cf. [1], [18], [26]). 
LEMmA 3.3. Let $E_{+}$be the forward fundamental solution for $\square$, and let $T \geq 2$. Assume that $T / 2 \leq t \leq T, 2^{-k-1} T \leq t-|x| \leq 2^{-k} T,|y| \leq s$, and $T / 10 \leq s$. Then

$$
|x /| x|-y /| y||^{2} \leq C 2^{-k} \text { if }(t, x, s, y) \in \operatorname{supp} E_{+}(t-s, x-y), 0 \leq k \leq \log _{2} T,
$$

for some uniform constant $C$.

Pr o of. We first recall the following version of Huygen's principle

$$
E_{+}(t-s, x-y)=0 \text { if }|x-y|>t-s .
$$

Because of this, our assumptions give

$$
E_{+}(t-s, x-y)=0 \text { if }|y|<s-2^{-k} T .
$$

Hence, assuming as we may that $k$ and $T$ are larger than some fixed constant, we must have $|x|,|y| \geq T / 20$ if $(t, x, s, y) \in \operatorname{supp} E_{+}(t-s, x-y)$ and the hypotheses of the lemma are fulfilled. For the final step, notice that $|x-y| \leq(t-s)^{2}$ is equivalent to

$$
\left|\frac{x}{|x|}-\frac{y}{|y|}\right|^{2} \leq \frac{(t-s)^{2}-(|x|-|y|)^{2}}{|x||y|}=\frac{(t-|x|-(s-|y|))(t+|x|-(s+|y|))}{|x||y|} .
$$

Since $|y| \leq s$, the right side is $\leq(t-|x|)(t+|x|) /|x||y|=O\left(2^{-k}\right)$, due to the fact that $t,|x|,|y| \approx T$ and $t-|x| \approx 2^{-k} T$ by our assumptions.

Using this lemma and Proposition 3.1 it is easy to prove (3.9). We simply need to exploit the fact that the weights involved are Lorentz-invariant and that our assumptions clearly imply that $t^{2}-|x|^{2} \geq 1$ if $(t, x) \in \operatorname{supp} F$.

To apply these observations we note that, since $w=E_{+} * F$, Lemma 3.3 implies that (3.9) would be a consequence of the further localized estimates

$$
\begin{aligned}
\left\|\left(t^{2}-|x|^{2}\right)^{-1 / 2+n(1 / 2-1 / q)} w\right\|_{L^{q}\left(\left\{(t, x): T / 2 \leq t \leq T, 2^{-k-1} T \leq t-|x| \leq 2^{-k} T,|x /| x|-\nu| \leq 2^{-k / 2}\right\}\right)} & \\
& \leq C(\log T)^{1 / q}\left\|\left(t^{2}-|x|^{2}\right)^{1 / q} F\right\|_{L^{q /(q-1)}},
\end{aligned}
$$

with $2 \leq q \leq 2(n+1) /(n-1), 0 \leq k \leq \log _{2} k$, and $\nu \in S^{n-1}$. The next thing to notice is that if we pick two points $\left(t_{j}, x_{j}\right), j=1,2$, in the support of the norm in the left, then we have the uniform bounds

$$
\operatorname{dist}\left(\left(\tilde{t}_{1}, \tilde{x}_{1}\right),\left(\tilde{t}_{2}, \tilde{x}_{2}\right)\right) \leq C
$$

if $(\tilde{t}, \tilde{x})=(t, x) / \sqrt{t^{2}-|x|^{2}}$ denotes the projection onto the unit hyperboloid in Lorentz space and $\operatorname{dist}((t, x),(s, y))=\sqrt{|x-y|^{2}-(t-s)^{2}}$ is the associated metric. Because of (3.11) and the preceding observations, (3.10) follows immediately from Proposition 3.1 if one makes a Lorentz rotation sending $\left(t_{1}, x_{1}\right)$ to $\left(\sqrt{t_{1}^{2}-\left|x_{1}\right|^{2}}, 0\right)$.

4. Estimates related to the Goursat problem. To finish our proof of (1.9) it suffices to show that we have uniform bounds of the form

$$
\begin{aligned}
& \left\|\left(t^{2}-|x|^{2}\right)^{-1 / 2+n(1 / 2-1 / q)-\varepsilon} w\right\|_{L^{q}\left(\left\{(t, x) \in \mathbb{R}_{+}^{1+n}: T / 2 \leq t \leq T\right\}\right)} \\
& \quad \leq C_{\varepsilon}\left\|\left(t^{2}-|x|^{2}\right)^{1 / q+\varepsilon} F\right\|_{L^{q /(q-1)}}, \quad 2 \leq q \leq 2(n+1) /(n-1), \quad \varepsilon>0,
\end{aligned}
$$

assuming now that

$$
F(t, x)=0 \text { if } t-|x|<1 \text { or } t>T / 10 \text {. }
$$


Since $\varepsilon$ is arbitrary and since $t^{2}-|x|^{2} \geq t$ in the norms, this inequality and (3.8) yield (1.9) if the parameter $\varepsilon$ there is larger than the one in (4.1).

This estimate is more delicate than (3.8) since, after scaling, we shall be forced to handle cases where $F$ is supported near the light cone and the norm on the left is taken over a region near the light cone containing characteristic rays emanating from the support of $F$. Because of this and the presence of the weights we cannot appeal to Proposition 2.1. Instead, we need to estimate certain degenerate Fourier integral operators which are similar to those arising in the solution of the Goursat problem.

Specifically, we shall require the following

Proposition 4.1. Suppose that

$$
f(y)=0 \text { if }|y| \notin[1,2] \text { or }|y /| y\left|-e_{1}\right| \geq c_{0} \delta^{1 / 2},
$$

where $e_{1}=(1,0,0, \ldots, 0)$. Then if $c_{0}>0$ is smaller than a uniform constant which is independent of $\delta<1$

$$
\int_{|\xi /| \xi\left|-e_{1}\right| \geq \delta^{1 / 2}}\left|\int e^{i y \cdot \xi-i|y||\xi|} f(y) d y\right|^{2} d \xi \leq C \delta^{-1}\|f\|_{L^{2}}^{2} .
$$

Proof. The proof is modeled after that of Proposition 2.1.

To start, we notice that by decomposing the conic region $\left\{\xi:|\xi /| \xi\left|-e_{1}\right| \geq \delta^{1 / 2}\right\}$ into a finite number of pieces, we see that it suffices to prove the estimate when we integrate over a convex conic subset $\Gamma_{\delta}$. Note then, for later use, that there is a uniform constant $C_{1}$ so that if $\delta<1$

$$
\left|\zeta^{\prime} /\right| \zeta|| \leq C_{1} \delta^{-1 / 2}\left|1-\zeta_{1} /\right| \zeta||, \zeta \in \Gamma_{\delta} .
$$

To be able to apply an integration by parts argument we need to make one further reduction. Specifically, suppose that $0 \leq a_{\delta} \in C^{\infty}$ is supported in the set where $1 / 2 \leq$ $|y| \leq 4$ and $|y /| y\left|-e_{1}\right| \leq 2 c_{0} \delta^{1 / 2}$ and satisfies the natural bounds

$$
\left|\left(\partial / \partial y_{1}\right)^{j}\left(\partial / \partial y^{\prime}\right)^{\alpha} a_{\delta}(y)\right| \leq C_{j, \alpha} \delta^{-|\alpha| / 2}, \forall j, \alpha,
$$

associated with this support assumption. Here $y^{\prime}=\left(y_{2}, \ldots, y_{n}\right)$. If we then set

$$
\left(S_{\delta} f\right)(\xi)=\int e^{i y \cdot \xi-i|y||\xi|} a_{\delta}(y) f(y) d y,
$$

then it suffices to show that

$$
\delta \int_{\xi \in \Gamma_{\delta}}\left|S_{\delta} f(\xi)\right|^{2} d \xi \leq C\|f\|_{L^{2}}^{2} .
$$

The dual version of this is equivalent to

$$
\delta\left\|S_{\delta} S_{\delta}^{*} h\right\|_{L^{2}\left(\Gamma_{\delta}\right)} \leq C\|h\|_{L^{2}}, \quad \text { supp } h \subset \Gamma_{\delta},
$$

where $S_{\delta} S_{\delta}^{*}$ is the integral operator with kernel

$$
K_{\delta}(\xi, \eta)=\int e^{i \Phi(y, \xi, \eta)} a_{\delta}^{2}(y) d y, \quad \xi, \eta \in \Gamma_{\delta},
$$

with the phase being

$$
\Phi(y, \xi, \eta)=y \cdot(\xi-\eta)-|y|(|\xi|-|\eta|) .
$$


Recall that $a_{\delta}(y)=0$ if $|y /| y\left|-e_{1}\right| \geq 2 c_{0} \delta^{1 / 2}$. Assuming, as we may, that $c_{0}$ is small enough, we claim that there is a constant $A$ so that, for every $N$,

$$
K_{\delta}(\xi, \eta) \leq C_{N}\left\{\begin{array}{l}
\delta^{(n-1) / 2}\left(1+\delta\left|\xi_{1}-\eta_{1}\right|\right)^{-N}, \text { if } \delta^{1 / 2}\left|\xi_{1}-\eta_{1}\right| \geq A\left|\xi^{\prime}-\eta^{\prime}\right| \\
\delta^{(n-1) / 2-N}\left(\delta^{-1}+\left|\xi^{\prime}-\eta^{\prime}\right|^{2}\right)^{-N}, \text { if } \delta^{1 / 2}\left|\xi_{1}-\eta_{1}\right| \leq A\left|\xi^{\prime}-\eta^{\prime}\right| .
\end{array}\right.
$$

This yields (4.7) by Young's inequality since for large $N$

$$
\begin{aligned}
& \delta^{(n-1) / 2} \int_{A\left|\xi^{\prime}-\eta^{\prime}\right| \leq \delta^{1 / 2}\left|\xi_{1}-\eta_{1}\right|}\left(1+\delta\left|\xi_{1}-\eta_{1}\right|\right)^{-N} d \xi \\
& \quad+\delta^{(n-1) / 2-N} \int_{\delta^{1 / 2}\left|\xi_{1}-\eta_{1}\right| \leq A\left|\xi^{\prime}-\eta^{\prime}\right|}\left(\delta^{-1}+\left|\xi^{\prime}-\eta^{\prime}\right|^{2}\right)^{-N} d \xi=O\left(\delta^{-1}\right) .
\end{aligned}
$$

To prove the first bound we need to integrate by parts with respect to $y$. To do so we note that, by the mean value theorem,

$$
\begin{aligned}
\left|\partial \Phi / \partial y_{1}\right| & =\left|\left(\xi_{1}-\eta_{1}\right)-y_{1} /\right| y|(|\xi|-|\eta|)| \\
& =\left.\left|\left(\xi_{1}-\eta_{1}\right)-y_{1} /\right| y|\cdot| \zeta\right|^{-1} \zeta \cdot(\xi-\eta) \mid \\
& \geq\left|\xi_{1}-\eta_{1}\right| \cdot\left|1-\zeta_{1} /\right| \zeta||-\left.|| \zeta\right|^{-1} \zeta^{\prime} \cdot\left(\xi^{\prime}-\eta^{\prime}\right) \mid,
\end{aligned}
$$

where $\zeta$ is a point on the line segment connecting $\xi$ and $\eta$. Since we are assuming that $\Gamma_{\delta}$ is convex we must have $\zeta \in \Gamma_{\delta}$ and so $\left|1-\zeta_{1} /\right| \zeta|| \geq c \delta$ for some uniform $c>0$. Therefore, if we let $A=2 C_{1}$, where $C_{1}$ is as in (4.4), we conclude that for $\xi, \eta \in \Gamma_{\delta}$ we must have

$$
\left|\partial \Phi / \partial y_{1}\right| \geq c \delta / 2 \cdot\left|\xi_{1}-\eta_{1}\right| \text { if } \delta^{1 / 2}\left|\xi_{1}-\eta_{1}\right| \geq A\left|\xi^{\prime}-\eta^{\prime}\right| .
$$

Since we also have

$$
\left|\left(\partial / \partial y_{1}\right)^{j} \Phi\right| \leq C_{j} \delta|\xi-\eta| \leq C_{j}^{\prime} \delta\left|\xi_{1}-\eta_{1}\right|, j \geq 2, y \in \operatorname{supp} a_{\delta}
$$

for such $\xi$ and $\eta$, the integration by parts argument that was used in the proof of Proposition 2.1 yields the first bounds in (4.8). The factor $\delta^{(n-1) / 2}$ comes from the support properties of the symbol.

The argument for the other bound in (4.8) is similar except now we must use our assumption that $a_{\delta}=0$ when $|y /| y\left|-e_{1}\right| \geq 2 c_{0} \delta^{1 / 2}$ with $c_{0}$ small. To do this, we first note that

$$
\begin{aligned}
\left|\nabla_{y^{\prime}} \Phi\right| & \geq\left|\xi^{\prime}-\eta^{\prime}\right|-\left|y^{\prime} /\right| y|| \cdot|| \xi|-| \eta|| \\
& \geq\left|\xi^{\prime}-\eta^{\prime}\right|-2 c_{0} \delta^{1 / 2}|\xi-\eta| .
\end{aligned}
$$

Hence if $\left|\xi_{1}-\eta_{1}\right| \leq A \delta^{-1 / 2}\left|\xi^{\prime}-\eta^{\prime}\right|$, where $A$ is the fixed constant chosen in the last step, we conclude that

$$
\left|\nabla_{y^{\prime}} \Phi\right| \geq\left|\xi^{\prime}-\eta^{\prime}\right| / 2, y \in \operatorname{supp} a_{\delta}
$$

if $c_{0}$ is small. Notice also that, because of our assumptions,

$$
\left|\left(\partial / \partial y^{\prime}\right)^{\alpha} \Phi\right| \leq C|\xi-\eta| \leq C^{\prime} \delta^{-1 / 2}\left|\xi^{\prime}-\eta^{\prime}\right| .
$$

Using these two bounds one obtains the other part of (4.8), which finishes the proof.

The preceding proposition will be used to handle relatively high frequency contributions to the solution of (1.4). To handle the low frequency parts of the Fourier integrals defining $w$ we shall need the following related, but simpler, result. 
Proposition 4.2. If $T \geq 1$ then

$$
\begin{aligned}
& \left\|\int e^{i x \cdot \xi-i|x||\xi|} \hat{f}(\xi) d \xi\right\|_{L^{2}(T / 2 \leq|x| \leq T)} \\
& \quad \leq C T^{1 / 2}\left(\|\hat{f}\|_{L^{2}(|\xi| \leq 1)}+\sum_{k=0}^{\infty} 2^{k / 2}\|\hat{f}\|_{L^{2}\left(2^{k} \leq|\xi| \leq 2^{k+1}\right)}\right) .
\end{aligned}
$$

Proof. If we change variables, we can write the left side as

$$
T^{-n / 2}\left\|\int e^{i x \cdot \xi-i|x||\xi|} \hat{f}(\xi / T) d \xi\right\|_{L^{2}(1 / 2 \leq|x| \leq 1)} .
$$

If, for fixed $1 / 2 \leq t \leq 1$, we apply the the Sobolev trace theorem to the function

$$
x \rightarrow \int e^{i x \cdot \xi-i t|\xi|} \hat{f}(\xi / T) d \xi
$$

we find that

$$
\begin{aligned}
& \int_{\theta \in S^{n-1}}\left|\int_{\mathbb{R}^{n}} e^{i t \theta \cdot \xi-i t|\xi|} \hat{f}(\xi / T) d \xi\right|^{2} d \theta \\
& \leq C\left(\|\hat{f}(\xi / T)\|_{L^{2}(|\xi| \leq 1)}+\sum_{j=0}^{\infty} 2^{j / 2}\|\hat{f}(\xi / T)\|_{L^{2}\left(2^{j} \leq|\xi| \leq 2^{j+1}\right)}\right)^{2} .
\end{aligned}
$$

If we now integrate over $1 / 2 \leq t \leq 1$, we conclude that the left side of the inequality in the statement of the lemma is dominated by

$$
\begin{aligned}
& T^{-n / 2}\left(\|\hat{f}(\xi / T)\|_{L^{2}(|\xi| \leq 1)}+\sum_{j=0}^{\infty} 2^{j / 2}\|\hat{f}(\xi / T)\|_{L^{2}\left(2^{j} \leq|\xi| \leq 2^{j+1}\right)}\right) \\
& \quad \leq T^{-n / 2}\left(T^{1 / 2}\|\hat{f}(\xi / T)\|_{L^{2}(|\xi| \leq T)}+\sum_{2^{j-1} \geq T} 2^{j / 2}\|\hat{f}(\xi / T)\|_{L^{2}\left(2^{j} \leq|\xi| \leq 2^{j+1}\right)}\right) \\
& \quad \leq 2 T^{1 / 2}\left(\|\hat{f}\|_{L^{2}(|\xi| \leq 1)}+\sum_{k=0}^{\infty} 2^{k / 2}\|\hat{f}(\xi)\|_{L^{2}\left(2^{k} \leq|\xi| \leq 2^{k+1}\right)}\right),
\end{aligned}
$$

as desired.

5. End of proof. We now must prove (4.1). Since $t^{2}-|x|^{2} \geq t$ on the supports and since $\varepsilon>0$ is arbitrary, it suffices to show that the inequality holds when $1 \leq 2^{j} \leq$ $t-|x| \leq 2^{j+1}$ and $1 \leq 2^{k} \leq t \leq 2^{k+1}$ in supp $F$. This special case yields (4.1) for a larger value of $\varepsilon$ if the constants are independent of $j$ and $k$.

If we take

$$
T_{0}=T / 2^{k} \geq 10, \text { and } 1 / T_{0} \leq \delta_{0}=2^{j-k} \leq 1,
$$

then the aforementioned localized inequalities are equivalent to the uniform bounds

$$
\begin{aligned}
2^{-4 k \varepsilon}\left\|\left(t^{2}-|x|^{2}\right)^{-1 / 2+n(1 / 2-1 / q)-\varepsilon} w\right\|_{L^{q}\left(\left\{(t, x): T_{0} / 2 \leq t \leq T_{0}\right\}\right)} \\
\quad \leq C_{\varepsilon}\left\|\left(t^{2}-|x|^{2}\right)^{1 / q+\varepsilon} F\right\|_{L^{q /(q-1)}}, 2 \leq q \leq 2(n+1) /(n-1),
\end{aligned}
$$

assuming now that

$$
F(t, x)=0 \text { if } t-|x| \notin\left[\delta_{0}, 2 \delta_{0}\right] \text { or } t \notin[1,2] .
$$


Note that the weights are essentially constant on the support of $F$. We can simplify the notation if we decompose the norm in the left side of the preceding inequality into ones over regions where $t-|x|$ and, hence, $t^{2}-|x|^{2}$ are essentially constant. Specifically, since $\delta \geq 1 / T_{0}$, we notice that (5.1) would be a consequence of the uniform bounds

$$
\begin{aligned}
& \left(\delta T_{0}\right)^{-1 / 2+n(1 / 2-1 / q)}\left(\delta / T_{0}\right)^{\varepsilon}\|w\|_{L^{q}\left(\left\{(t, x): T_{0} / 2 \leq t \leq T_{0}, \delta \leq t-|x| \leq 2 \delta\right\}\right)} \\
& \leq C_{\varepsilon} \delta_{0}^{1 / q}\|F\|_{L^{q /(q-1)}}, 2 \leq q \leq 2(n+1) /(n-1), \delta_{0} \leq \delta \leq T_{0} .
\end{aligned}
$$

If $\delta>10$, say, the inequality is easy. For then $E_{+}(t-s, x-y)=O\left((t(t-|x|))^{-(n-1) / 2}\right)$ for $(t, x)$ in the support of the norm in the left and $(s, y) \in \operatorname{supp} F$. Therefore, the estimate for $\delta>10$ just follows from Hölder's inequality, and so from now on we shall assume that

$$
\delta_{0} \leq \delta \leq 10 \text {. }
$$

As before, by interpolation, to prove (5.3), it suffices to show that the inequality holds for the endpoints where $q=2$ or $q=2(n+1) /(n-1)$. The arguments for the two cases are slightly different. Let us first concentrate on $q=2(n+1) /(n-1)$, since this is the more delicate of the two cases.

For this exponent, let us notice that it is easy to handle (5.3) when $\delta \approx \delta_{0}$. Indeed, since

$$
-1 / 2+n(1 / 2-1 / q)=1 / q, \text { when } q=2(n+1) /(n-1),
$$

a stronger version of (5.3) for, say, $\delta_{0} \leq \delta \leq 10 \delta_{0}$ would be

$$
T_{0}^{1 / q}\|w\|_{L^{q}\left(\left\{(t, x): T_{0} / 2 \leq t \leq T_{0}\right\}\right)} \leq C\|F\|_{L^{q /(q-1)}}, 2(n+1) /(n-1) .
$$

But if we use a routine freezing argument (see, e.g. [19, §0.3]), we see that this follows from the following estimates of Strichartz [25]

$$
\|u(t-s, \cdot)\|_{L^{q}\left(\mathbb{R}^{n}\right)} \leq C|t-s|^{-2 / q}\|g\|_{L^{q /(q-1)}\left(\mathbb{R}^{n}\right)},
$$

where

$$
u(t, x)=(2 \pi)^{-n} \int e^{i x \cdot \xi} \sin (t|\xi|) \hat{g}(\xi) d \xi /|\xi| .
$$

This inequality implies the preceding one since if we let $K(t, s)=|t-s|^{-2 / q}$, when $(t, s) \in\left[T_{0} / 2, T_{0}\right] \times[1,2]$ and 0 otherwise then, by Hölder's inequality, the associated integral operator sends $L^{q /(q-1)}(\mathbb{R})$ to $L^{q}(\mathbb{R})$ with norm $O\left(T_{0}^{-1 / q}\right)$.

To handle the remaining cases where $10 \delta_{0} \leq \delta \leq 10$, first notice that if we use Hölder's inequality, as in the proof of Proposition 3.1, then we find that $w$ in (5.3) is dominated by $\delta_{0}^{1 / q}$ times

$$
\left(\left.\left.\int_{\delta_{0}}^{2 \delta_{0}}\left|\iint e^{i(x-y) \cdot \xi}\right| \xi\right|^{-1} \sin ((t-\tau-|y|)|\xi|) F(\tau+|y|, y) d \xi d y\right|^{q /(q-1)} d \tau\right)^{(q-1) / q} .
$$

Therefore, since we are assuming that $\delta \geq 10 \delta_{0}$, if we replace $t$ by $t-\tau$, we conclude that the remaining cases of (5.3) for $q=2(n+1) /(n-1)$ would be a consequence of the following

Proposition 5.1. For $n \geq 2$ set

$$
(W g)(t, x)=\int_{\mathbb{R}^{n}} \int_{\left\{y \in \mathbb{R}^{n}: 1 \leq|y| \leq 2\right\}} e^{i(x-y) \cdot \xi-i(t-|y|)|\xi|} g(y) d y d \xi /|\xi| .
$$


Then, if $q=2(n+1) /(n-1), \varepsilon>0, t>5$ and $\delta<10$

$$
\|W g(t, \cdot)\|_{L^{q}(\{x: \delta \leq t-|x| \leq 2 \delta\})} \leq C t^{\varepsilon-2 / q} \delta^{-\varepsilon-1 / q}\|g\|_{L^{q /(q-1)}} .
$$

As before, we shall prove this using complex interpolation. To this end, let us set

$$
\left(W_{z} g\right)(t, x)=(z-(n+1) / 2) e^{z^{2}} \iint_{1 \leq|y| \leq 2} e^{i(x-y) \cdot \xi-i(t-|y|)|\xi|}|\xi|^{-z} g(y) d y d \xi
$$

so that $W_{1}$ is a multiple of $W$. Therefore, if we apply complex interpolation we conclude that (5.5) would be a consequence of

$$
\left\|W_{z} g(t, \cdot)\right\|_{L^{\infty}\left(\mathbb{R}^{n}\right)} \leq C t^{-(n-1) / 2}\|g\|_{L^{1}}, \text { Re } z=(n+1) / 2,
$$

and

$$
\left\|W_{z} g(t, \cdot)\right\|_{L^{2}(\{x: \delta \leq t-|x| \leq 2 \delta\})} \leq C t^{\varepsilon / 2} \delta^{-\varepsilon-1 / 2}\|g\|_{L^{2}}, \text { Re } z=0 .
$$

Inequality (5.6) follows immediately from (1.12) and our assumption that $t \geq 5$. The $L^{2}$ estimate is much harder. For it we shall need to use ideas from microlocal analysis. These techniques will only allow us to handle the contributions from large frequencies $\xi$, depending on the scales $\delta$ and $t$. Fortunately, though, it is easy to handle low frequencies by appealing to Proposition 4.2 .

To be more precise, let

$$
\alpha=1+\varepsilon / 2,
$$

and fix $\rho \in C^{\infty}$ satisfying $\rho(\xi)=0$ for $|\xi| \leq 1$ and $\rho=1$ for $|\xi| \geq 2$. If we then let

$$
\begin{aligned}
& \left(R_{z} g\right)(t, x) \\
& =(z-(n+1) / 2) e^{z^{2}} \iint_{1 \leq|y| \leq 2} e^{i(x-y) \cdot \xi-i(t-|y|)|\xi|}|\xi|^{-z}\left(1-\rho\left(t^{1-\alpha} \delta^{\alpha} \xi\right)\right) g(y) d y d \xi,
\end{aligned}
$$

the dual version of (4.10) corresponding to $T=2$ yields

$$
\left\|R_{z} g(t, \cdot)\right\|_{L^{2}\left(\mathbb{R}^{n}\right)} \leq C t^{(\alpha-1) / 2} \delta^{-\alpha / 2}\|g\|_{L^{2}\left(\mathbb{R}^{n}\right)}, \text { Re } z=0 .
$$

In view of this, we conclude that (5.7) would follow if

$$
\left\|S_{z} g(t, \cdot)\right\|_{L^{2}(\{x: \delta \leq t-|x| \leq 2 \delta\})} \leq C \delta^{-1 / 2}\|g\|_{L^{2}}, \text { Re } z=0
$$

with

$$
\left(S_{z} g\right)(t, x)=e^{z^{2} / 2} \iint_{1 \leq|y| \leq 2} e^{i(x-y) \cdot \xi-i(t-|y|)|\xi|}|\xi|^{-z} \rho\left(t^{1-\alpha} \delta^{\alpha} \xi\right) g(y) d y d \xi .
$$

Note that the bounds here are stronger than those in the two preceding inequalities. However, since we shall need to apply Proposition 4.1, it is necessary to assume here that $t-|x|$ is bounded below by $\delta$ in the norm in the left.

To proceed we need a couple of lemmas. Both follow from straightforward integration by parts arguments (see [3]).

LEMMA 5.2. If $a(\xi)$ belongs to a bounded subset of $S^{0}$, and if $\rho \in C^{\infty}$ satisfies $\rho(\xi)=0$ for $|\xi| \leq 1$, and $\rho=1,|\xi| \geq 2$, then, for $\alpha>1$ and $t>1$,

$$
\left|\int e^{i x \cdot \xi-i t|\xi|} a(\xi) \rho\left(t^{1-\alpha} \delta^{\alpha} \xi\right) d \xi\right| \leq C_{N, \alpha}(\delta / t)^{N}, \quad \text { if }|| x|-t| \geq \delta / 2 .
$$


Lemma 5.3. Suppose that $\psi(\tau) \in C^{\infty}(\mathbb{R})$ vanishes near $\tau=0$ and equals 1 when $|\tau|$ is large. Then if $\delta$ is small, $\rho$ and $\alpha>1$ are as above, and $t>1$,

$$
\left|\int e^{i x \cdot \xi-i t|\xi|} a(\xi) \psi\left(\delta^{-1 / 2}(x /|x|-\xi /|\xi|)\right) \rho\left(t^{1-\alpha} \delta^{\alpha} \xi\right) d \xi\right| \leq C_{N}(\delta / t)^{N},
$$

where, for a given $N$, the constants depend only on dist(0, supp $\psi)$ and the size of finitely many derivatives of $\psi$, if $\rho$ is fixed and a belongs to a bounded subset of $S^{0}$.

To apply these two lemmas we require another simple geometric lemma. In contrast to Lemma 3.3, which concerned the intersection properties of two essentially externally tangent spheres, the following is about the intersection of two essentially internally tangent spheres.

Lemma 5.4. Suppose that $t>5,1 \leq|y| \leq 2$ and that ||$x-y|-| t-|y||| \leq \delta / 2$ and $\delta \leq t-|x| \leq 2 \delta$. It then follows that if $\delta$ is smaller than a fixed positive constant

$$
|y /| y|-x /| x|| \in\left[C_{0}^{-1} \delta^{1 / 2}, C_{0} \delta^{1 / 2}\right]
$$

for some absolute constant $C_{0}$.

Proof. As in the proof of Lemma 3.3 we shall use the identity

$$
\left|\frac{x}{|x|}-\frac{y}{|y|}\right|^{2}=\frac{|x-y|^{2}-(|x|-|y|)^{2}}{|x||y|}=\frac{|x-y|+|x|-|y|}{|x||y|} \cdot \frac{|x-y|-(|x|-|y|)}{\delta} \cdot \delta .
$$

By our assumptions the first factor on the right is bounded from above and below. Writing $|x-y|-(|x|-|y|)=|x-y|-(t-|y|)+t-|x|$, we reach the same conclusion for the second factor, yielding the result.

To use Lemma 5.4 we note that if $1 \leq|y| \leq 2,|x| \geq 4, \nu \in S^{n-1}$ and $|x /| x|-\nu| \geq \delta^{1 / 2}$ then $|(x-y) /| x-y|-\nu| \geq \delta^{1 / 2} / 2$ if $\delta$ is small and $|y /| y|-\nu| \leq c_{0} \delta^{1 / 2}$, with $c_{0}>0$ being a small uniform constant. With this in mind, we conclude that, for small $\delta,(5.9)$ follows from Proposition 5.1. Thus we have established the inequalities when $\delta<\delta_{1}$, with $\delta_{1}$ being a uniform small constant.

Clearly, though, the above arguments will also show that (5.9) holds if $\delta_{1}<\delta<10$. To see this we note that, by an integration by parts argument, if $t-|x| \geq \delta_{1}$ and $\operatorname{Re} z=0$ we can write

$$
\begin{aligned}
& \left(S_{z} g\right)(t, x) \\
& =e^{z^{2} / 2} \iint_{1 \leq|y| \leq 2} e^{i(x-y) \cdot \xi-i(t-|y|)|\xi|}|\xi|^{-z} \rho\left(t^{1-\alpha} \delta^{\alpha} \xi\right) b(y /|y|-\xi /|\xi|) g(y) d y d \xi+O\left(t^{-N}\right),
\end{aligned}
$$

if $b \in C^{\infty}$ vanishes near $\xi=0$ but equals one outside of a sufficiently small neighborhood the origin (depending on $\delta_{1}$ ). Because of this, the estimate for $\delta>\delta_{1}$ just follows from Proposition 2.1.

To finish, we still need to see that (5.3) holds when $q=2$, assuming (5.2) and (5.4). To do this, as in the preceding case, it is convenient to split things into a low and high frequency part. To this end, let us write $w=w_{0}+w_{1}$, where

$$
w_{0}=\iint e^{i(x-y) \cdot \xi} \beta(\delta \xi) \sin ((t-s)|\xi|) F(s, y) d s d y d \xi /|\xi|
$$


where $\beta \in C_{0}^{\infty}$ satisfying $\beta=1$ near the origin has been fixed. It then suffices to show that when $q=2,(5.3)$ holds when $w$ is replaced by $w_{j}, j=0,1$.

Since $(1-\beta(\delta \xi)) /|\xi|=O(\delta)$, the bound for the high frequency part just follows from Schwarz's inequality and the variant of (5.7) where $|\xi|^{-z}$, Re $z=0$ is replaced by $\delta^{-1}(1-\beta(\delta \xi)) /|\xi|$. Since this inequality clearly follows from the proof of (5.7), we are left with estimating the low frequency part $w_{0}$.

For this piece, we first note that

$$
\int_{|\xi| \leq 1} e^{i(x-y) \cdot \xi} \beta(\delta \xi) \sin ((t-s)|\xi|) d \xi /|\xi|=O\left((1+|x-y|)^{-(n-1) / 2}\right) .
$$

Because of this, we conclude that the variant of (5.3) holds when $q=2$ if we replace $w$ by

$$
\iint_{|\xi| \leq 1} e^{i(x-y) \cdot \xi} \beta(\delta \xi) \sin ((t-s)|\xi|) F(s, y) d s d y d \xi /|\xi| .
$$

Consequently, our proof of (5.3) and hence (1.9) would be complete if we could show that when

we have

$$
\tilde{w}(t, x)=\iint_{|\xi| \geq 1} e^{i(x-y) \cdot \xi+i(t-s)|\xi|}|\xi|^{-1} \beta(\delta \xi) F(s, y) d s d y d \xi
$$

(5.10) $T_{0}^{-1 / 2} \delta^{-1 / 2}\|\tilde{w}\|_{L^{2}\left(\left\{(t, x): T_{0} / 2 \leq t \leq T_{0}, \delta \leq t-|x| \leq 2 \delta\right\}\right)} \leq C(1+|\log \delta|) \delta_{0}^{1 / 2}\|F\|_{L^{2}}$

To prove this, we first notice that the Schwarz inequality and Proposition 4.2 give

$$
\begin{aligned}
& T_{0}^{-1 / 2} \delta^{-1 / 2}\|\tilde{w}\|_{L^{2}\left(\left\{(t, x): T_{0} / 2 \leq t \leq T_{0}, \delta \leq t-|x| \leq 2 \delta\right\}\right)} \\
& \quad \leq C \sum_{k=0}^{\infty}\left(\left.\left.\int\left|\iint_{2^{k} \leq|\xi| \leq 2^{k+1}} e^{i(x-y) \cdot \xi-i s|\xi|}\right| \xi\right|^{-1 / 2} \beta(\delta \xi) F(s, y) d \xi d s d y\right|^{2} d x\right)^{1 / 2} .
\end{aligned}
$$

If we recall (5.2) and use Schwarz's inequality as before, we see that the right side is dominated by $\delta_{0}^{1 / 2}$ times

$$
\sum_{k=0}^{\infty}\left(\left.\left.\iint\left|\iint_{2^{k} \leq|\xi| \leq 2^{k+1}} e^{i(x-y) \cdot \xi-i(\tau+|y|)|\xi|}\right| \xi\right|^{-1 / 2} \beta(\delta \xi) F(\tau+|y|, y) d \xi d y\right|^{2} d x d \tau\right)^{1 / 2}
$$

yielding (5.10) by the dual version of Proposition 4.2 since the $k$-th summand vanishes if $k$ is larger than a fixed multiple of $(1+|\log \delta|)$.

6. Further problems. It would be interesting to try to sharpen the inequalities stated here in a number of ways. First, one would like to have a scale invariant version of (1.9). For odd spatial dimensions, in the spherically symmetric case, it was shown in [3] that if $F=0$ for $|x|>t$ and if $w$ solves (1.4), then

$$
\begin{aligned}
& \left\|\left(t^{2}-|x|^{2}\right)^{-\alpha} w\right\|_{L^{q}\left(\mathbb{R}_{+}^{1+n}\right)} \leq C_{\gamma}\left\|\left(t^{2}-|x|^{2}\right)^{\beta} F\right\|_{L^{q /(q-1)}\left(\mathbb{R}_{+}^{1+n}\right)}, \\
& \quad \text { if } \beta<1 / q, \quad \alpha+\beta+\gamma=2 / q, \quad \text { where } \gamma=(n-1)(1 / 2-1 / q),
\end{aligned}
$$

provided that $2<q \leq 2(n+1) /(n-1)$. Presumably this inequality extends to the general case. In view of the discussion in the introduction, such an inequality would be sharp.

It would also be interesting to try to prove a natural weighted Strichartz inequality which does not involve assuming a lower bound on $t-|x|$ in the support of $F$. Such 
estimates might allow one to drop the assumption that the data in (1.1) have compact support and replace it by sharp decay assumptions at infinity (cf. [12]).

Finally, it would be interesting to obtain sharp lifespan estimates for subcritical powers $p<1$, that is, those satisfying

$$
(n-1) p^{2}-(n+1) p-2 \leq 0 .
$$

In [12], sharp estimates were obtained if $1<p \leq(n+1) /(n-1)$. However, it is not clear that the techniques in the present paper can be used to obtain sharp results for larger subcritical powers.

\section{References}

[1] J. Bourgain, Averages in the plane over convex curves and maximal operators, J. Analyse Math. 47 (1986), 69-85.

[2] G. Eskin, Degenerate elliptic pseudo-differential operators of principal type (Russian), Mat. Sbornik 82 (124) (1970), 585-628; English translation, Math. USSR Sbornik 11 (1970), 539-582.

[3] V. Georgiev, H. Lindblad and C. D. Sogge, Weighted Strichartz estimates and global existence for semilinear wave equations, Amer. J. Math., to appear.

[4] R. Glassey, Finite-time blow-up for solutions of nonlinear wave equations Math. Z. 177, 323-340.

[5] R. Glassey, Existence in the large for $\square u=F(u)$ in two dimensions, Math. Z 178 (1981), 233-261

[6] J. Harmse, On Lebesgue space estimates for the wave equation, Indiana Math. J. 39 (1990), 229-248.

[7] L. Hörmander, Fourier integral operators I, Acta Math. 127 (1971), 79-183.

[8] D. Jerison and C. E. Kenig, Unique continuation and absence of positive eigenvalues for Schrödinger operators, Annals of Math. 121 (1985), 463-494.

[9] F. John, Blow-up of solutions of nonlinear wave equations in three space dimensions, Manuscripta Math. 28 (1979), 235-265.

[10] H. Kubo, On the critical decay and power for semilinear wave equations in odd space dimensions, preprint.

[11] H. Lindblad and C. D. Sogge, On existence and scattering with minimal regularity for semilinear wave equations, J. Funct. Anal. 130 (1995), 357-426.

[12] H. Lindblad and C. D. Sogge, Long-time existence for small amplitude semilinear wave equations, Amer. J. Math. 118 (1996), 1047-1135.

[13] H. Lindblad and C. D. Sogge, Restriction theorems and semilinear Klein-Gordon equations in (1+3)-dimensions, Duke Math. J. 85 (1996), 227-252.

[14] C. Müller, On the behavior of the solutions of the differential equation $\Delta U=F(x, U)$ in the neighborhood of a point, Comm. Pure Appl. Math. 7 (1954), 505-514.

[15] J. Schaeffer, The equation $u_{t t}-\Delta u=|u|^{p}$ for the critical value of $p$, Proc. Royal Soc. Edinburgh 101 (1985), 31-44.

[16] A. Seeger, C. D. Sogge and E. M. Stein, Regularity properties of Fourier integral operators, Annals of Math. 134 (1985), 231-251.

[17] T. Sideris, Nonexistence of global solutions of wave equations in high dimensions, Comm. Partial Diff. Equations 12 (1987), 378-406. 
[18] C. D. Sogge, Propagation of singularities and maximal functions in the plane, Invent. Math. 104 (1991), 349-376.

[19] C. D. Sogge, Fourier integrals in classical analysis, Cambridge Tracts in Math. 105, Cambridge Univ. Press, Cambridge, 1993.

[20] C. D. Sogge, Lectures on nonlinear wave equations, International Press, Cambridge, 1995.

[21] C. D. Sogge and E. M. Stein, Averages of functions over hypersurfaces: Smoothness of generalized Radon transforms, J. Analyse Math. 54, 165-188.

[22] E. M. Stein, Interpolation of linear operators, Trans. Amer. Math. Soc. 83 (1956), 482 492.

[23] W. Strauss, Nonlinear scattering theory, Scattering theory in mathematical physics, Reidel, Dordrect, 1979, pp. 53-79.

[24] W. Strauss, Nonlinear scattering at low energy, J. Funct. Anal. 41 (1981), 110-133.

[25] R. Strichartz, A priori estimates for the wave equation and some applications, J. Funct. Analysis 5 (1970), 218-235.

[26] T. Wolff, A sharp $L^{3}$ estimate via incidence geometry, Amer. J. Math. (to appear).

[27] Y. Zhou, Cauchy problem for semilinear wave equations with small data in four space dimensions, J. Diff. Equations 8 (1995), 135-144. 